\title{
Assessing the Readjustable Sling Procedure (Remeex System) for Female Stress Urinary Incontinence With Detrusor Underactivity
}

\author{
Kwang Jin Ko ${ }^{1}$, Yoon Seok Suh ${ }^{1}$, Hyun Hwan Sung ${ }^{1}$, Gyu Ha Ryu², Munjae Lee ${ }^{3}$, Kyu-Sung Lee ${ }^{1,3}$ \\ ${ }^{1}$ Department of Urology, Samsung Medical Center, Sungkyunkwan University School of Medicine, Seoul, Korea \\ ${ }^{2}$ Office of R\&D Strategy \& Planning, Samsung Medical Center, Seoul, Korea \\ ${ }^{3}$ Department of Medical Device Management \& Research, SAIHST, Sungkyunkwan University, Seoul, Korea
}

Purpose: Many surgeons worry about postoperative voiding problems in patients with stress urinary incontinence (SUI) and detrusor underactivity (DU). This study hypothesized that adjusting the tension after surgery would benefit patients with DU, and therefore researchers evaluated the outcomes and quality of life of women with SUI and DU who underwent the readjustable sling procedure (Remeex).

Methods: The medical records of 27 patients who were treated with the Remeex system for SUI and DU between 2007 and 2013 were retrospectively analyzed. The incontinence visual analogue scale (I-VAS), incontinence quality of life questionnaire (I-QOL) and the Sandvik incontinence severity index (ISI) were used to evaluate the efficacy of the Remeex system both prior to surgery and at the last visit after surgery. The treatment was considered successful if there was no urine leakage based on the Sandvik questionnaire.

Results: The mean follow-up period was 38.0 months (range, 1-75 months), and the treatment success rate was $81.5 \%$. Despite no urine leakage, 7 patients wanted the Remeex system to be removed due to persistent postoperative urinary retention within a year of surgery. One patient underwent a long-term adjustment under local anesthesia 6 years after the initial surgery. The I-VAS, ISI and all domains of the I-QOL scores improved significantly postoperatively and the maximum flow rate decreased significantly after the procedure. However, the postvoid residual did not change significantly.

Conclusions: The Remeex system provided a good cure rate and improved the quality of life of women with SUI and DU. Therefore, the Remeex system should be considered as a treatment option for female patients with concomitant SUI and DU.

Keywords: Female; Midurethral Sling; Urinary Incontinence, Stress

- Fund/Grant Support: This research was supported by a grant of the Korea Health Technology R\&D Project through the Korea Health Industry Development Institute (KHIDI), funded by the Ministry of Health \& Welfare, Republic of Korea (grant number: HI14C3229).

- Research Ethics: This study was performed with the approval of the Institutional Review Board of Samsung Medical Center at Sungkyunkwan

University School of Medicine (approval number: 2015-04-098).

- Conflict of Interest: No potential conflict of interest relevant to this article was reported.

\section{INTRODUCTION}

Stress urinary incontinence (SUI) is predominantly a female health problem that affects approximately $7.9 \%-46.0 \%$ of wom- en, and increases in prevalence with age [1,2]. Several studies have found that midurethal slings (MUS) have excellent longterm effectiveness with a relatively low complication rate [3-5]. However, despite the development of several MUS methods,

Corresponding author: Kyu-Sung Lee (iD https://orcid.org/0000-0003-0891-2488 Department of Urology, Samsung Medical Center, Sungkyunkwan University School of Medicine, 50 Irwon-ro, Gangnam-gu, Seoul 06351, Korea E-mail: ksleedr@skku.edu / Tel: +82-2-3410-3554 / Fax: +82-2-3410-3027 Submitted: July 16, 2016 / Accepted after revision: September 5, 2016

c) (P) This is an Open Access article distributed under the terms of the Creative Commons Attribution Non-Commercial License (http://creativecommons.org/licenses/by-nc/4.0/) which permits unrestricted non-commercial use, distribution, and reproduction in any medium, provided the original work is properly cited. 
the optimum tension to apply when the sling is placed beneath the midurethra is still controversial. Excessive tension on the urethra may cause postoperative bladder outlet obstruction. A multi-institutional comparison of obstructive voiding complications between transobturator and transabdominal MUS demonstrated that the transobturator approach is associated with fewer obstructive complications than is the transabdominal approach (transabdominal, 18.3\%; transobturator, 11.0\%) [6]. After the transobturator tape procedure, complete urinary retention occurred in $0 \%-13.3 \%$ of patients, while mesh cutting or adjustment was required in up to $5 \%$ of cases [5].

If patients have SUI with detrusor underactivity (DU) as confirmed by a urodynamic study (UDS), many surgeons worry about the development of postobstructive voiding problems. In addition, there are no clear treatment guidelines for SUI with DU.

This study hypothesized that adjusting the tension after surgery would benefit patients with DU. Several methods have been used to control the tension postoperatively, such as transobturator adjustable tape (TOA) and the readjustable sling procedure (Remeex, Regulation Mechanical Eternal; Neomedic International, Terrassa, Spain) [7-10]. There have been no known studies of Remeex system in treatment for SUI with pure DU, and therefore, the outcomes and complications of the Remeex system for SUI and DU in women were evaluated in this study.

\section{MATERIALS AND METHODS}

The study was a retrospective analysis of the medical records of 27 patients who met the inclusion criteria of primary SUI with DU and underwent the Remeex procedure between January 2007 and April 2013 at single center. For the purpose of the study, DU was defined as a contraction of reduced strength and/or duration that results in prolonged bladder emptying and/or a failure to empty completely within a reasonable time span, as referenced in the 2002 International Continence Society (ICS) standardization report. [11].

At baseline, all of the patients were evaluated based on their medical history, physical examination including a stress test (cough provocation), uroflowmetry (UFM), postvoid residual (PVR), and multichannel UDS. The urodynamic evaluation consisted of filling and voiding cystometry, abdominal leak point pressure, detrusor pressure at the time of maximum flow, and maximal detrusor pressure measurements. All of the defi- nitions and methods correspond to those of ICS. In addition, the Sandvik incontinence severity index (ISI) [12], which measures the severity of incontinence symptoms, the incontinence quality of life (I-QOL) survey [13], which assesses incontinence-specific quality of life, and the incontinence visual analogue scale (I-VAS) were used.

A single experienced surgeon performed all of the Remeex procedures. The Remeex device consists of polypropylene mesh with 2 nonabsorbable sutures and a regulation device (varitensor). The surgery was performed under anesthesia with the patient in the dorsal lithotomy position and a 20F Foley catheter was inserted. First, an anterior vaginal wall incision was made at the midurethral level and the underlying periurethral tissues were dissected. A 3- to 4-cm transverse incision was then made in the suprapubic area, and the sutures were passed to the suprapubic incision site by a suture passer needle driven by a passer handle. The same procedure was carried out on the opposite side. After that, cystoscopic examination was performed to confirm bladder integrity and the suspension suture was inserted into the varitensor. The mesh was then placed at the midurethra and the manipulator was wound clockwise until the varitensor was approximately 2 fingerbreadths above the rectus fascia. When the varitensor was positioned appropriately, the incision site was sutured.

On the first postoperative day, the Foley catheter was removed and the tension was adjusted by rotating the manipulator based on the patient stress test and UFM. All patients were evaluated 1 week postoperatively followed by $1,3,6$, and 12 months postoperatively, using the following: I-VAS, ISI, and IQOL questionnaires and UFM parameters. The treatment was considered successful if there was no urine leakage during coughing or physical activities based on the Sandvik questionnaire at the final follow-up. Any adverse events were also evaluated.

Descriptive statistics were used to evaluate patient characteristics, and Wilcoxon's rank sum test was conducted as appropriate. All data analyses were performed using IBM SPSS Statistics ver. 20.0 (IBM Co., Armonk, NY, USA). Statistical significance was set at $\mathrm{P}<0.05$.

\section{RESULTS}

The mean follow-up period was 38.0 months (range, 1-75 months). Table 1 lists the baseline characteristics of the $27 \mathrm{pa}-$ tients who underwent the Remeex procedure. Patients had sev- 
eral etiologic factors contributing to DU, such as previous radical pelvic surgeries, which comprised 16 patients (59.3\%) who underwent radical hysterectomy due to cervical cancer and 1 patient (3.7\%) who underwent the Miles operation for rectal cancer. Other factors included 3 patients (11.1\%) with diabetes mellitus, 2 with spinal lesions (7.4\%) (severe lumbar spine spon-

Table 1. Demographic data of 27 patients who underwent a readjustable sling procedure (Remeex) for female stress urinary incontinence with detrusor underactivity

\begin{tabular}{lc}
\hline Variable & Value \\
\hline Age $(\mathrm{yr})$ & $59.0(51-70)$ \\
Body mass index $\left(\mathrm{kg} / \mathrm{m}^{2}\right)$ & $25.56 \pm 3.12$ \\
No. of vaginal deliveries, median (range) & $2(1-9)$ \\
Mixed incontinence & $2(7.4)$ \\
Previous anti-incontinence surgery & \\
Tension-free suburethral sling operation & $4(14.8)$ \\
Bulking agent injection & $5(18.5)$ \\
Urodynamic study parameters & \\
ALPP $\left(\mathrm{cm} \mathrm{H}_{2} \mathrm{O}\right)$ & $92.1 \pm 36.8$ \\
PdetQmax $\left(\mathrm{cm} \mathrm{H}_{2} \mathrm{O}\right)$ & $17.4 \pm 13.6$ \\
MaxPdet $\left(\mathrm{cm} \mathrm{H}_{2} \mathrm{O}\right)$ & $24.3 \pm 16.1$ \\
Maximal flow rate $(\mathrm{mL} / \mathrm{sec})$ & $12.6 \pm 6.3$ \\
Postvoid residual $(\mathrm{mL})$ & $72.1 \pm 88.8$ \\
\hline
\end{tabular}

Values are presented as median (interquartile rarnge), mean \pm standard deviation, or number (\%).

ALPP, abdominal leak point pressure; PdetQmax, detrusor pressure at the time of maximum flow; MaxPdet, maximal detrusor pressure. dylosis and lumbo-sacral lipoma), and 1 with Parkinson disease (3.7\%). Four patients (14.8\%) had idiopathic DU (Table 2).

Only 1 of the 27 enrolled patients was lost to follow-up. The treatment success was $81.5 \%$ ( 22 of 26). There were 7 patients who had no urine leakage after surgery, but requested that the Remeex system be removed due to persistent postoperative urinary retention within a year. One patient underwent a longterm adjustment under local anesthesia 6 years after the surgery. Among the treatment failure patients (4 of 26), 3 patients refused further readjustment, and 1 underwent another midurethral sling (Miniarc, American Medical Systems, Minnetonka, MI, USA) surgery 5 months after the initial surgery.

In the subgroup analysis of 18 patients who had completed IVAS, ISI, and I-QOL questionnaires before surgery and 1 year postsurgery, there was significant improvement in I-VAS $(\mathrm{P}<$ 0.001 ). In addition, the mean total score of the I-QOL questionnaire increased $(\mathrm{P}=0.004)$. The mean avoidance and limiting behavior $(\mathrm{P}=0.004)$, psychosocial impact $(\mathrm{P}=0.006)$, and so-

Table 2. Etiologic factors leading to detrusor underactivity

\begin{tabular}{lc}
\hline Etiologic factor & No. $(\%)$ \\
\hline Previous pelvic surgery due to malignancy & $17(59.3)$ \\
Idiopathic & $4(14.8)$ \\
Diabetes mellitus & $3(11.1)$ \\
Spine lesion & $2(7.4)$ \\
Parkinson disease & $1(3.7)$ \\
\hline
\end{tabular}

Table 3. Changes in symptom severity as evaluated by questionnaire and uroflowmetry parameters in patients before and after the Remeex procedure

\begin{tabular}{|c|c|c|c|}
\hline Variable & Baseline & Postoperation & P-value \\
\hline I-VAS & $7.6 \pm 2.3$ & $3.1 \pm 2.7$ & $<0.001$ \\
\hline Sandvik ISI & & & 0.001 \\
\hline None & 0 & 6 & \\
\hline Slight & 1 & 3 & \\
\hline Moderate & 0 & 3 & \\
\hline Severe & 9 & 5 & \\
\hline Very severe & 8 & 1 & \\
\hline \multicolumn{4}{|l|}{$\mathrm{I}-\mathrm{QOL}$} \\
\hline Total I-QOL score & $64.8 \pm 90.2$ & $146.8 \pm 115.2$ & 0.004 \\
\hline Avoidance and limiting behaviors & $23.2 \pm 32.8$ & $51.2 \pm 38.2$ & 0.004 \\
\hline Psychosocial impacts & $24.1 \pm 29.9$ & $51.3 \pm 39.5$ & 0.006 \\
\hline Social embarrassment & $17.5 \pm 29.3$ & $44.1 \pm 39.7$ & 0.009 \\
\hline Maximal flow rate (mL/sec) & $12.6 \pm 6.3$ & $8.9 \pm 5.7$ & 0.044 \\
\hline Postvoid residual (mL) & $72.1 \pm 88.8$ & $56.8 \pm 87.5$ & 0.717 \\
\hline
\end{tabular}

Values are presented as mean \pm standard deviation or number.

I-VAS, incontinence visual analogue scale; ISI, incontinence severity index; I-QOL, incontinence quality of life scale. 
cial embarrassment $(\mathrm{P}=0.009)$ domain scores also improved significantly after surgery. The maximum flow rate (MFR) decreased significantly after the procedure; however, the PVR did not change significantly (Table 3 ). Nine patients (50\%) experienced I-QOL increases of $>10$ points after surgery, which represents the minimal important difference for I-QOL [14]. The MFR decreased significantly $(\mathrm{P}=0.044)$ after the procedure, but the PVR did not change significantly $(\mathrm{P}=0.717)$ (Table 3$)$.

There were no serious complications in this study. Bladder injury occurred intraoperatively in 1 case (3.7\%). Postoperatively, complications included 1 case (3.7\%) of de novo urgency, 7 cases $(25.9 \%)$ of persistent urinary retention, and 2 cases (7.4\%) of wound infection. The Remeex system was removed after 40 months in the case of wound infection.

\section{DISCUSSION}

This is the first study to demonstrate the benefits and high treatment success rate (81.5\%) of the Remeex system on female SUI with DU. Kim and Kim [15] described that DU cannot influence the surgical outcomes of midurethral sling for SUI with an $88 \%$ success rate. In addition, only a few articles exist on the TOA procedure for SUI with concomitant voiding difficulty. In a study by Jo et al. [16], patients with DU and intrinsic sphincter deficiency were included; however, DU was simply defined as MFR $<15 \mathrm{~mL} / \mathrm{sec}$. The group reported an objective complete cure rate of $60.0 \%$ (18 patients) and a $33.3 \%$ improvement rate (10 patients). Lee et al. [17] performed a follow-up in 65 women who underwent TOA due to severe SUI and combined SUI and voiding difficulty (MFR $\leq 12 \mathrm{~mL} / \mathrm{sec}$ with a void volume $\geq 100 \mathrm{~mL}$ ). At 6 months, this group showed an $84.4 \%$ complete cure rate for SUI and an $86.2 \%$ patient satisfaction rate. Unlike former studies using either midurethral sling or adjustable sling, which defined DU rather simply, this study defined DU more comprehensively based on patients' voiding symptoms, straining pattern of UFM, large PVR and/or relationship between detrusor pressure and urine flow. This study was conducted in real patients with underlying DU who were suspected of having a high risk of voiding difficulty due to conditions such as previous radical pelvic surgery or neurogenic etiology. In that sense, these data demonstrate that the Remeex system is an excellent treatment option for patients with both SUI and DU. In addition, with the Sandvik ISI, the total score and the scores for all domains of the I-QOL significantly improved after surgery in this study (all, $\mathrm{P}<0.05$ ).
The original purpose of using Remeex was to improve incontinence while maintaining voiding function. It is particularly difficult to balance the functions of voiding and storage in patients with SUI and DU. Low tension might sustain the same voiding level as that prior to surgery, without relieving the urinary incontinence. In this study, 3 persistent SUI patients who failed the initial treatment refused to have additional tension adjustment for personal reasons, such as old age or bedridden status. However, all of them had the possibility of readjustment of tension and their degrees of incontinence improved subjectively.

In contrast, high tension may resolve incontinence, but may also cause voiding difficulties. In these cases, clean intermittent catheterization (CIC) becomes necessary. Some patients with DU may find that voiding difficulties (with higher tension) are more inconvenient than is urinary incontinence. Seven patients who experienced complete resolution of their SUI symptoms failed to void even when the tension was loosened maximally during the postoperative follow-up period (range, 1-22 weeks). CIC was recommended, but was declined. Therefore, the Remeex system was removed in all of these cases.

The initial cases of the surgery were performed while leaving a certain amount of tension during the operation. On the first postoperative day, the tension was loosened while also taking into consideration the voiding symptoms and incontinent levels of the patients. The patients that removed the Remeex system due to voiding difficulties were all in initial cases. Since then, the Remeex procedure was conducted without applying any tension during the operation and the following day, the tension was tightened to calibrate an optimal status between voiding difficulties and level of incontinence. Afterwards, there were no more cases of removal of the Remeex due to discomfort related to voiding difficulties.

A few studies have evaluated the risk factors for urinary retention after the MUS procedure. Hong et al. [18] demonstrated that predictive factors after the tension-free vaginal tape procedure were mean age, parity, MFR, and a history of hysterectomy. MFR was the only independent factor in the multivariate analysis. In the present study, statistically predictive factors of urinary retention could not be found. However, there was a high rate of a history of radical hysterectomy in the persistent urinary retention group. This finding suggests that patients who have SUI and DU, especially those who previously underwent radical hysterectomies, should be warned of their higher risk of requiring lifelong CIC. 
This study was based on retrospective, noncomparative analysis. One limitation of the study was the small number of patients included. Statistical verification of the relationship between operative success and the cause of DU was not possible. In addition, clinically objective tests to evaluate the treatment success of the Remeex system were not available. Finally, the UDS parameter cutoffs used to define DU cannot be explicitly stated.

In conclusion, the Remeex system is an effective treatment option for patients with concomitant female SUI and DU with a success rate of $81.5 \%$. Although SUI symptoms may fluctuate and recur, readjustment of tension can be easily implemented whenever necessary. Further large-scale studies with long-term follow-up are needed. Regardless, it was found that the Remeex system improved quality of life in women with SUI and DU.

\section{REFERENCES}

1. Botlero R, Urquhart DM, Davis SR, Bell RJ. Prevalence and incidence of urinary incontinence in women: review of the literature and investigation of methodological issues. Int J Urol 2008;15:2304.

2. Kwon CS, Lee JH. Prevalence, risk factors, quality of life, and health-care seeking behaviors of female urinary incontinence: results from the 4th Korean National Health and Nutrition Examination Survey VI (2007-2009). Int Neurourol J 2014;18:31-6.

3. Ulmsten U, Petros P. Intravaginal slingplasty (IVS): an ambulatory surgical procedure for treatment of female urinary incontinence. Scand J Urol Nephrol 1995;29:75-82.

4. Delorme E. Transobturator urethral suspension: mini-invasive procedure in the treatment of stress urinary incontinence in women. Prog Urol 2001;11:1306-13.

5. Schimpf MO, Rahn DD, Wheeler TL, Patel M, White AB, Orejuela FJ, et al. Sling surgery for stress urinary incontinence in women: a systematic review and metaanalysis. Am J Obstet Gynecol 2014; 211:71.e1-71.e27.

6. Morey AF, Medendorp AR, Noller MW, Mora RV, Shandera KC, Foley JP, et al. Transobturator versus transabdominal mid urethral slings: a multi-institutional comparison of obstructive voiding complications. J Urol 2006;175(3 Pt 1):1014-7.

7. Campos-fernandes JL, Timsit MO, Paparel P, Devonec M, Leriche B, Leriche A, et al. REMEEX: A possible treatment option in selected cases of sphincter incompetence. Prog Urol 2006;16:184-91.
8. Errando C, Rodriguez-Escovar F, Gutierrez C, Baez C, Araño P, Villavicencio $\mathrm{H}$. A re-adjustable sling for female recurrent stress incontinence and sphincteric deficiency: outcomes and complications in 125 patients using the Remeex sling system. Neurourol Urodyn 2010;29:1429-32.

9. Giberti C, Gallo F, Cortese P, Schenone M. The suburethral tension adjustable sling (REMEEX system) in the treatment of female urinary incontinence due to 'true' intrinsic sphincter deficiency: results after 5 years of mean follow-up. BJU Int 2011;108:1140-4.

10. Mantovani F, Castelnuovo C, Bernardini P. ReMeEx device (External Mechanical Regulator) for incontinence: implantation and regulation procedure, complications and results at 3 years follow-up. Arch Ital Urol Androl 2004;76:49-50.

11. Abrams P, Cardozo L, Fall M, Griffiths D, Rosier P, Ulmsten U, et al. The standardisation of terminology of lower urinary tract function: report from the Standardisation Sub-committee of the International Continence Society. Neurourol Urodyn 2002;21:167-78.

12. Sandvik H, Hunskaar S, Seim A, Hermstad R, Vanvik A, Bratt H. Validation of a severity index in female urinary incontinence and its implementation in an epidemiological survey. J Epidemiol Community Health 1993;47:497-9.

13. Oh SJ, Park HG, Lim SH, Hong SK, Martin ML, Ting BL, et al. Translation and linguistic validation of Korean version of the incontinence quality of life (I-QoL) instrument. J Korean Continence Soc 2002;6:10-23.

14. Patrick DL, Martin ML, Bushnell DM, Marquis P, Andrejasich CM, Buesching DP. Cultural adaptation of a quality-of-life measure for urinary incontinence. Eur Urol 1999;36:427-35.

15. Kim SJ, Kim JC. Influence of preoperative detrusor underactivity on the continence rate and satisfaction after midurethral sling patient with stress urinary incontinence. Low Urin Tract Symptoms 2010;2:95-9.

16. Jo DG, Yang SA, Seo JT. Effects of transobturator adjustable tape sling procedure on the therapeutic outcome in patients with stress urinary incontinence and detrusor underactivity. Int Neurourol J 2010;14:20-5.

17. Lee SY, Lee YS, Lee HN, Choo MS, Lee JG, Kim HG, et al. Transobturator adjustable tape for severe stress urinary incontinence and stress urinary incontinence with voiding dysfunction. Int Urogynecol J 2011;22:341-6.

18. Hong B, Park S, Kim HS, Choo MS. Factors predictive of urinary retention after a tension-free vaginal tape procedure for female stress urinary incontinence. J Urol 2003;170:852-6. 